Stud. Univ. Babeş-Bolyai Math. 65(2020), No. 2, 309-320

DOI: $10.24193 /$ subbmath.2020.2.12

\title{
Nonlinear economic growth dynamics in the context of a military arms race
}

\author{
Daniel Metz and Adrian Viorel
}

\begin{abstract}
In the present contribution, we propose and analyze a dynamical economic growth model for two rival countries that engage an arms race. Under natural assumptions, we prove that global solutions exist and discuss their asymptotic long-time behavior. The results of our stability analysis support the recurring hypothesis in Cold War political science that engaging in an arms race with a technologically superior and hence faster growing adversary has damaging economic consequences. Numerical findings illustrate our claims.
\end{abstract}

Mathematics Subject Classification (2010): 91B62, 91F10.

Keywords: Solow-Swan model, arms race, asymptotic behavior.

\section{Introduction}

The Cold War has ended three decades ago and regional antagonisms have replaced the previous colossal struggle. Nevertheless, our understanding of the United States vs. USSR Arms Race and its wider economic consequences remains far from complete (see [7]).

A plausible, and often repeated explanation attributes the Eastern Block's collapse to an economic crisis triggered by unsustainable military ambitions. Given the ever growing and ever more visible gap in technological and economic capabilities, matching American military development was possible only at the expense of economic growth and stability.

The aim of the present contribution is to examine this hypothesis from an analytic point of view by developing a model that, at least qualitatively, reproduces economic stagnation caused by a prolonged military rivalry with a faster developing adversary.

Arms races have a long history that goes back far beyond the Cold War Era. The ancient Greeks and Romans built fleets to match their Persian and Carthaginian rivals, but the naval race that followed the 1889 Naval Defense Act calling for the Royal 
Navy to be as strong as the world's next two largest navies combined, is probably the most intensively studied predecessor as it led to World War I (WWI).

For decades, Arms races have been a topical subject in Political Science, such that the scarcity of treatments from a mathematical perspective comes as a real surprise - all the more so given L. F. Richardson's pioneering contributions to the field synthesized in Arms and Insecurity: A Mathematical Study of the Causes and Origins of War [12] and Statistics of Deadly Quarrels [13].

The classical Richardson model, which relies on a system of two coupled linear differential equations, has dominated theoretical debates for more than half a century. If $x(t)$ and $y(t)$ denote the levels of arms for two rival states, with rates of change driven by the sum of a positive reaction to the other country's arms, a negative 'fatigue' reaction to own military level and a constant 'grievance' term, then the time evolution is described by

$$
\left\{\begin{array}{l}
\frac{\mathrm{d} x}{\mathrm{~d} t}(t)=-\beta_{11} x(t)+\beta_{12} y(t)+\gamma_{1} \\
\frac{\mathrm{d} y}{\mathrm{~d} t}(t)=\beta_{21} x(t)-\beta_{22} y(t)+\gamma_{2} .
\end{array}\right.
$$

The unique equilibrium point of the system, which exists provided that the two straight lines defined by the right hand side of (1.1) are not parallel, may be unstable and Richardson related exponentially diverging solutions with the outbreak of war. Nonlinear extensions of the classical Richardson model have been considered by Hill [6].

We take a similar approach but augment the model by adding an economic dimension described in terms of Solow-Swan dynamics discussed below based on [3]. A different line of thought, that we don't pursue here, deals with arms races or, more generally, strategic interactions from a game theoretical perspective. Two or more actors play a (repeated) game in which the strategies that they can choose from are to arm or not to arm (see, for example [10]). For a recent contribution that is somewhat pertaining to the present work, we refer to [9]. The direction contrary to our study, that is disarmament models has also been pursued (cf. [4]), while a strongly misleading use of the term arms race in a biological context has been rendered popular by Dawkins and Krebs in [5].

The Solow-Swan model, originating from the independent works [14] and [15], explains long-run economic growth in a neoclassical framework by relating capital, labor and technology. The model relies on three fundamental assumptions. The first assumption is an exponential population (or labor) growth

$$
L(t)=L_{0} e^{n t} \quad\left(\text { equivalently } \frac{\mathrm{d} L}{\mathrm{~d} t}=n L\right) .
$$

The second assumption concerns a Cobb-Douglas production function connecting the economic output $Y$ to the labor $L$, capital $K$ and the level of technology $A$

$$
Y=A K^{\alpha} L^{1-\alpha} \text {. }
$$

Here, $\alpha \in[0,1]$ is the returns to scale constant. 
The third assumption of the model asserts that change in capital $K(t)$ is due to the positive capital output saving (with saving rate $\sigma \in(0,1)$ ) and to the negative capital depreciation (at a rate $\delta$ )

$$
\frac{\mathrm{d} K}{\mathrm{~d} t}=\sigma Y-\delta K
$$

By combining these three assumptions and expressing them in terms of the capital intensity

$$
k(t)=\frac{K(t)}{L(t)}
$$

one obtains

$$
\frac{\mathrm{d} k}{\mathrm{~d} t} L+k n L=\frac{\mathrm{d} K}{\mathrm{~d} t}=\sigma A(k L)^{\alpha} L^{1-\alpha}-\delta(k L)
$$

and deduces the fundamental equation of the Solow-Swan model

$$
\frac{\mathrm{d} k}{\mathrm{~d} t}=\sigma A k^{\alpha}-(n+\delta) k .
$$

Observe that here we have reached an explicitly solvable Bernoulli equation which defines a dynamical system with two equilibria, $k^{*}=0$ being unstable in contrast to

$$
k^{*}=\left(\frac{\sigma A}{n+\delta}\right)^{1 / 1-\alpha}
$$

which is asymptotically stable (attractor).

\section{An economic growth model with arms race military expenses}

The simplicity and lack of specificity proved to be both a strength and weakness of the classical Richardson model which has become a cornerstone of strategic thinking despite the somewhat imprecise concept of arms not allowing rigorous fitting to measurable data. It turns out that replacing weapon quantities by an abstract 'security' concept that can be linked to economic factors is more lucrative. Loosely following discrete models in both Krabs [8] and Larrosa [9] we consider an augmented arms race model in underlying economic growth context

$$
\left\{\begin{aligned}
\frac{\mathrm{d} s_{1}}{\mathrm{~d} t}(t) & =-k_{2}(t) s_{1}(t)+k_{1}(t) s_{2}(t) \\
\frac{\mathrm{d} s_{2}}{\mathrm{~d} t}(t) & =k_{2}(t) s_{1}(t)-k_{1}(t) s_{2}(t) \\
\frac{\mathrm{d} k_{1}}{\mathrm{~d} t}(t) & =a_{1} k_{1}(t)^{\alpha}-b k_{1}(t)-c s_{2}(t) k_{1}(t) \\
\frac{\mathrm{d} k_{2}}{\mathrm{~d} t}(t) & =a_{2} k_{2}(t)^{\alpha}-b k_{2}(t)-c s_{1}(t) k_{2}(t) .
\end{aligned}\right.
$$

Here, $s_{i}(t)$ describes the level of security of the state $i$ at time $t, k_{i}(t)$ being the country's capital intensity. Security levels obey Richardson-type equations but with 
time-varying coefficients. The competitive nature of the model is reflected in the fact that an increase in one actor's security is its adversary's security loss as

$$
\frac{\mathrm{d} s_{1}}{\mathrm{~d} t}(t)=-\frac{\mathrm{d} s_{2}}{\mathrm{~d} t}(t)
$$

In other words the total security is constant $s_{1}(t)+s_{2}(t)=$ const and imposing $s_{1}(0)+s_{2}(0)=1$ will assure, as we will see in the next section, $0 \leq s_{1}(t), s_{2}(t) \leq 1$ meaning that security levels range from 0 (totally insecure) to 1 (totally secure).

On the other hand, both economies grow according to a Solow-Swan model with an additional term explicitly accounting for military expenses. These exchange terms including the adversary's security might look surprising at first glance, but in view of (2.2) one country's security is the other's insecurity $s_{j}=1-s_{i}$ and military expenses are proportional precisely to the insecurity $1-s_{i}$. The coefficient $c \in(0,1)$ represents a budget constraint and expresses the percentual limit which military spendings cannot exceed in a functional peacetime economy.

Returning to the security equations, one can now see that the right hand side terms are actually proportional to military costs, insecurity rising based on rival spending and decreasing based on own spendings.

The parameters $\alpha, b$ and $a_{i}$ retain their original Solow model meaning and only $a_{1}, a_{2}$ differ from country to country. In view of (1.2), this difference is essential to our model and accounts for the technological gap separating the two economies.

\section{Analysis of the model}

We start our analysis by discussing an uncoupled Solow-Swan model with variable military expenditures. Quite naturally, the best and worst case scenarios, namely zero or maximal military spending, provide upper and lower bounds for the dynamics.

Lemma 3.1 (upper and lower bounds). Let us consider the initial value problem

$$
\frac{\mathrm{d} k}{\mathrm{~d} t}=a k^{\alpha}-b k-c s(t) k, \quad k(0)=k_{0}
$$

with coefficients $a>0, \alpha \in(0,1), b>0, c \in[0,1]$ and $s:[0, \infty) \rightarrow \mathbb{R}$ a given smooth function with $s(t) \in[0,1]$ for any $t \geq 0$. If $k_{0}>0$ then the solution of $(3.1)$ exists, is positive and satisfies for all times

a) $k(t) \leq \bar{k}(t)$, where $\bar{k}$ is the solution of

$$
\frac{\mathrm{d} \bar{k}}{\mathrm{~d} t}=a \bar{k}^{\alpha}-b \bar{k}, \quad \bar{k}(0)=k_{0}
$$

a) $\underline{k}(t) \leq k(t)$, where $\underline{k}$ is the solution of

$$
\frac{\mathrm{d} \underline{k}}{\mathrm{~d} t}=a \underline{k}^{\alpha}-b \underline{k}-c \underline{k}, \quad \underline{k}(0)=k_{0} .
$$

Proof. In (3.1) we are dealing with a Bernoulli equation which is exactly solvable, hence the global existence using the usual change substitution $z(t)=k(t)^{1-\alpha}$. Using 
the variation of constants formula one has the desired positivity from

$$
k(t)^{1-\alpha}=z(t)=e^{-\int_{0}^{t} \frac{b+c s(\rho)}{1-\alpha} \mathrm{d} \rho}\left[k_{0}^{1-\alpha}+\frac{a}{1-\alpha} \int_{0}^{t} e^{\int_{0}^{\tau} \frac{b+c s(\rho)}{1-\alpha} \mathrm{d} \rho} \mathrm{d} \tau\right] .
$$

To obtain both the upper and lower bounds, one can rely on standard sub and supersolution arguments. Since $0 \leq s(t) \leq 1$ and $k(t) \geq 0$

$$
a k^{\alpha}-b k-c k \leq \frac{\mathrm{d} k}{\mathrm{~d} t} \leq a k^{\alpha}-b k
$$

and the conclusion follows.

Remark 3.2. From a dynamical systems point of view, both autonmous equations in Lemma 3.1 are Solow-Swan equations and have the same stability behavior albeit with different nonzero asymptotically stable equilibria namely

$$
\bar{k}^{*}=\left(\frac{a}{b}\right)^{\frac{1}{1-\alpha}} \text { and } \quad \underline{k}^{*}=\left(\frac{a}{b+c}\right)^{\frac{1}{1-\alpha}}
$$

respectively.

After this helpful preliminaries we are in position to prove the existence of global solutions to $(2.1)$.

Theorem 3.3 (global existence). Let us consider the growth under arms race rivalry model (2.1) with $a_{1}, a_{2}>0, \alpha \in(0,1), b>0$ and $c \in[0,1]$. Then for any initial conditions $k_{1}(0), k_{2}(0)>0$ and $s_{1}(0), s_{2}(0)>0$ with $s_{1}(0)+s_{2}(0)=1$ there exists a unique classical solution of the initial value problem associated to the system (2.1) which remains bounded for all $t \geq 0$.

Proof. We divide the proof in several steps.

Step 1. Local existence. As the right hand side of the system has good regularity (only local Lipschitz continuity is actually required), a standard Banach fixed point argument guarantees the existence of local in time solutions, defined on a maximal interval $t \in[0, T), T=T\left(s_{1}(0), s_{2}(0), k_{1}(0), k_{2}(0)\right.$.

Step 2. Positivity of $k_{1}$ and $k_{2}$. Based on the representation formula (3.4) which holds on their maximal interval of existence $t \in[0, T)$, one can see that for positive initial states $k_{1}(0), k_{2}(0)>0$, both $k_{1}(t)$ and $k_{2}(t)$ must be positive for $t \in[0, T)$.

Step 3. Positivity of $s_{1}$ and $s_{2}$. Using the fact that

$$
s_{1}(t)+s_{2}(t)=1 \quad \text { for all } t \in[0, T),
$$

one can rewrite the evolution equations for $s_{1}$ and $s_{2}$ as

$$
\begin{aligned}
\frac{\mathrm{d} s_{1}}{\mathrm{~d} t} & =k_{1}(t)-\left(k_{1}(t)+k_{2}(t)\right) s_{1}, \\
\frac{\mathrm{d} s_{2}}{\mathrm{~d} t} & =k_{2}(t)-\left(k_{1}(t)+k_{2}(t)\right) s_{2},
\end{aligned}
$$

such that aplying the variation of constants formula one again

$$
s_{1}(t)=e^{-\int_{0}^{t}\left(k_{1}(\rho)+k_{2}(\rho)\right) \mathrm{d} \rho}\left[s_{1}(0)+\int_{0}^{t} e^{\int_{0}^{\tau}\left(k_{1}(\rho)+k_{2}(\rho)\right) \mathrm{d} \rho} k_{1}(\tau) \mathrm{d} \tau\right]
$$


which is positive for positive $k_{1}$. Similarly, one can show that $s_{2}$ has the same property. In view of (3.5) and the positivity of $s_{1}, s_{2}$ we have

$$
0 \leq s_{1}(t), s_{2}(t) \leq 1 \quad \text { for all } t \in[0, T)
$$

Step 4. Boundedness of $k_{1}, k_{2}$ and global solutions. The estimates in (3.8) not only assure the boundedness for $s_{1}, s_{2}$ but also allow us to apply Lemma 3.1, more precisely the upper bound in a), and hence deduce the boundedness of $k_{1}$ and $k_{2}$. A classical result (see Barbu [2]) now assures that the local in time solutions can be extend to arbitrary positive times.

\section{Asymptotic behavior of the model}

We start by determining the equilibrium points of the system, that is the solutions of

$$
\left\{\begin{array}{l}
0=-k_{2}^{*} s_{1}^{*}+k_{1}^{*} s_{2}^{*}, \\
0=k_{2}^{*} s_{1}^{*}-k_{1}^{*} s_{2}^{*} \\
0=a_{1}\left(k_{1}^{*}\right)^{\alpha}-b k_{1}^{*}-c s_{2}^{*} k_{1}^{*} \\
0=a_{2}\left(k_{2}^{*}\right)^{\alpha}-b k_{2}^{*}-c s_{1}^{*} k_{2}^{*} .
\end{array}\right.
$$

One can reduce this to a 3 by 3 nonlinear system by assuming that (3.5) holds. The resulting equilibrium equations are

$$
\left\{\begin{array}{l}
0=k_{1}^{*}-\left(k_{1}^{*}+k_{2}^{*}\right) s_{1}^{*} \\
\left.0=a_{1}\left(k_{1}^{*}\right)^{\alpha}-b k_{1}^{*}-c(1-) s_{1}^{*}\right) k_{1}^{*} \\
0=a_{2}\left(k_{2}^{*}\right)^{\alpha}-b k_{2}^{*}-c s_{1}^{*} k_{2}^{*} .
\end{array}\right.
$$

Trivial equilibria, that is with $k_{i}^{*}=0$, exist but are not interesting from a modeling perspective as they would indicate the disappearance of an economy. Nevertheless, we note without going into details, that all such equilibria are unstable, as a natural consequence of the lower bound b) in Lemma 3.1 means that both $k_{1}$ and $k_{2}$ are pushed away from zero even when starting arbitrarily close.

However, there exists also a nontrivial equilibrium point.

\subsection{The unique nontrivial equilibrium}

In terms of the convenient notation

$$
R^{*}=\frac{s_{1}^{*}}{s_{2}^{*}}=\frac{k_{1}^{*}}{k_{2}^{*}},
$$

from (3.5) we have

$$
s_{1}^{*}=\frac{R^{*}}{1+R^{*}} \quad \text { and } \quad s_{2}^{*}=\frac{1}{1+R^{*}} .
$$

Such that inserting this in the $3^{r d}$ and $4^{\text {th }}$ equation of (4.1) leads to

$$
\left(\frac{k_{1}^{*}}{k_{2}^{*}}\right)^{1-\alpha}=\frac{a_{1}}{a_{2}} \cdot \frac{b+(b+c) R^{*}}{(b+c)+b R^{*}},
$$


that is,

$$
\left(R^{*}\right)^{1-\alpha}=\frac{a_{1}}{a_{2}} F\left(R^{*}\right)
$$

with

$$
F(R)=\frac{b+(b+c) R}{(b+c)+b R} .
$$

Hence, finding the nontrivial equilibrium reduces to solving the coincidence problem (4.4) (or equivalently the fixed point problem $R^{*}=\frac{a_{1}}{a_{2}} F\left(R^{*}\right)^{1 / 1-\alpha}$ ). The existence of a unique nontrivial coincidence point $R^{*}$ follows from geometric considerations.

Lemma 4.1. The coincidence problem (4.4) with $a_{1} \geq a_{2}$ has a unique solution $R^{*} \geq 1$ provided that $\alpha \in\left[0, \frac{1}{2}\right)$ and $\frac{c}{2 b+c} \leq 1-\alpha$. If $a_{1}>a_{2}$ then $R^{*}>\frac{a_{1}}{a_{2}}$.

Proof. We start by noting some geometric properties of $F$. One can easily check that

$$
F(0)=\frac{b}{b+c}, \quad F(1)=1 \quad \text { and } \quad F(\infty)=\frac{b+c}{b}
$$

while

$$
F^{\prime}(R)=\frac{(2 b+c) c}{((b+c)+b R)^{2}}>0 \quad \text { and } \quad F^{\prime \prime}(R)<0 .
$$

In other words, $F$ is monotonically increasing, convex and bounded from above by $b+c / b$. Consequently, the range of $R \mapsto \frac{a_{1}}{a_{2}} F(R)$ is $\left[\frac{a_{1}}{a_{2}} \frac{b}{b+c}, \frac{a_{1}}{a_{2}} \frac{b+c}{b}\right]$.

Now, observe that for $\frac{a_{1}}{a_{2}}=1, R=1$ is a solution of

$$
R^{1-\alpha}=F(R)
$$

For the moment, we assume that there are no other solutions in $(0,1)$ and later give a sufficient condition for this to hold true.

If $R^{1-\alpha}=F(R)$ has no solutions is $(0,1)$, that is, $F(R)>R^{1-\alpha}$ for $R \in(0,1)$ due to $F(0)=b /(b+c)>0$, then $\frac{a_{1}}{a_{2}} R^{1-\alpha}=F(R)$ has no solutions in $\left(0, a_{1} / a_{2}\right)$. Indeed, on one hand

$$
R^{1-\alpha}<F(R) \leq \frac{a_{1}}{a_{2}} F(R) \quad \text { for } \quad 0<R<1
$$

while on the other hand

$$
R^{1-\alpha}<R<\frac{a_{1}}{a_{2}}<\frac{a_{1}}{a_{2}} F(R) \quad \text { for } \quad 1<R<\frac{a_{1}}{a_{2}} .
$$

As $R \mapsto R^{1-\alpha}$ is increasing and unbounded while $F$ is increasing and bounded, the two curves will cross at a unique point $R^{*}>\frac{a_{1}}{a_{2}}$.

The necessary and sufficient condition for $R^{1-\alpha}=F(R)$ to have no solutions in $(0,1)$ is again geometric in nature. Actually, the slope of $F$ at $R=1$ must not exceed that of $G(R)=R^{1-\alpha}$, that is precisely

$$
\frac{c}{2 b+c} \leq 1-\alpha
$$


Remark 4.2. Both essential conditions for the uniqueness of the coincidence point

$$
\alpha<\frac{1}{2} \text { and } \quad \frac{c}{2 b+c} \leq 1-\alpha
$$

are natural and in accordance with econometric data. The returns to scale constant is generally considered to be $\alpha \approx 1 / 3$ while the depreciation constant is $b \approx 0.05$ (see Acemoglu [1]). On the other hand, even at the hight of the Cold War, according to the World Bank ${ }^{1}$ military expenses have not exceeded $10 \%$ of GDP, so roughly $c \approx 2 b$, which satisfies the coincidence condition.

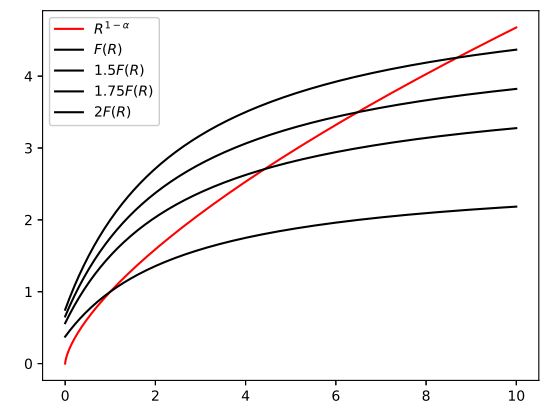

Figure 1. Qualitative behavior of the coincidence problem under the uniqueness assumptions (4.5).

In the sequel, we analyze the stability of the equilibrium point corresponding to this unique $R^{*}$, that is of (4.3) together with

$$
k_{1}^{*}=\left(\frac{a_{1}}{b+\frac{c}{1+R^{*}}}\right)^{\frac{1}{1-\alpha}} \text { and } \quad k_{2}^{*}=\left(\frac{a_{2}}{b+\frac{c R^{*}}{1+R^{*}}}\right)^{\frac{1}{1-\alpha}} .
$$

To this end, we compute the Jacobi matrix of the (3 by 3 ) system which is

$$
J\left(s_{1}^{*}, k_{1}^{*}, k_{2}^{*}\right)=\left[\begin{array}{ccc}
-\left(k_{1}^{*}+k_{2}^{*}\right) & 1-s_{1}^{*} & -s_{1}^{*} \\
c k_{1}^{*} & T_{1} & 0 \\
-c k_{2}^{*} & 0 & T_{2}
\end{array}\right]
$$

with

$$
T_{1}=(\alpha-1) \frac{b+c+b R^{*}}{1+R^{*}} \quad \text { and } \quad T_{2}=(\alpha-1) \frac{b+(b+c) R^{*}}{1+R^{*}} .
$$

We discuss the eigenvalues of this matrix in two different, parameter-dependent cases.

\footnotetext{
${ }^{1}$ https://data.worldbank.org/indicator/MS.MIL.XPND.GD.ZS?locations=US
} 


\subsection{The catch-up scenario $a_{1}=a_{2}=a$}

This is the simpler yet less realistic situation in which there exists no difference between the parameters describing the two countries, this especially means that both economies have the same technology level, and only their initial states may differ.

Returning to (4.4), one can see that it reduces to the simpler

$$
\left(R^{*}\right)^{1-\alpha}=F\left(R^{*}\right)
$$

which has the unique coincidence point $R^{*}=1$. As a consequence

$$
s_{1}^{*}=s_{2}^{*}=\frac{1}{2} \quad \text { and } \quad k_{1}^{*}=k_{2}^{*}=k^{*}=\left(\frac{a}{b+\frac{1}{2} c}\right)^{\frac{1}{1-\alpha}}
$$

and straightforward but rather tedious computation show that all three eigenvalues of the Jacobian $J\left(\frac{1}{2}, k^{*}, k^{*}\right)$ have negative real part, so the equilibrium is locally asymptotically stable.

From a modeling perspective, this describes a catch-up evolution in which the country with the initially weaker economy will recover the deficit in the long-run and stabilize at the same level as its rival, as depicted in Figure 2.
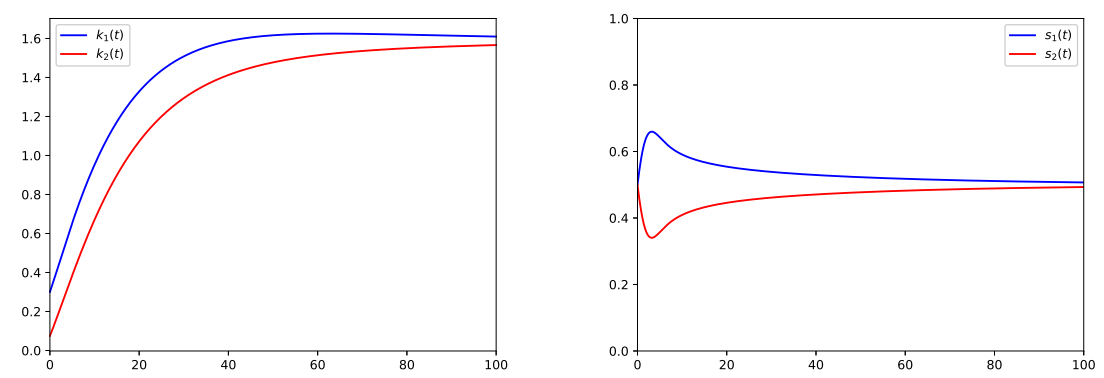

Figure 2. The catch-up scenario $a_{1}=a_{2}$. The two countries experience a convergent economic growth with the initialy weaker economy catching up to the stronger (Left panel). Security levels also converge towards a balanced stationary state (Right panel). Simulations correspond to $a_{1}=a_{2}=0.15, \alpha=1 / 3, b=0.06, c=0.1$ and $s_{1}(0)=s_{2}(0)=1 / 2, k_{1}(0)=0.3, k_{2}(0)=k_{1}(0) / 4$.

\subsection{The increasing gap scenario $a_{1}>a_{2}$}

From our point of view, the more interesting and realistic situation is that of unequal coefficients $a_{1}>a_{2}$. This describes a technological gap between the two contenders, and we will show that the quotient $a_{1} / a_{2}$ plays a decisive role in the long-term dynamics as its affects the equilibrium quotient $R^{*}$ of the two economies (see Figure 3). 

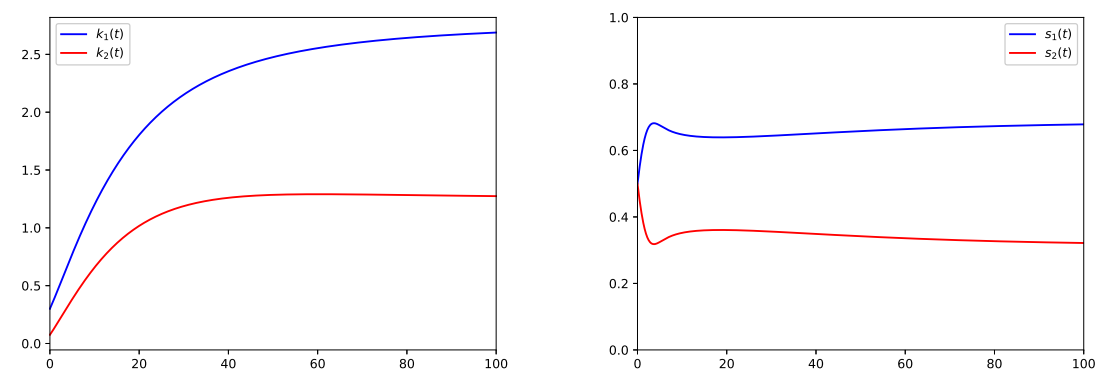

Figure 3. The increasing gap scenario $a_{1}>a_{2}$. Despite growth for both countries, the gap separating them widens as excessive military spending harms the slower developing one (Left panel). Security levels also separate in the stronger economy's favour (Right panel). Simulations correspond to $a_{1}=0.18, a_{2}=0.15, \alpha=1 / 3, b=0.06, c=0.1$ and $s_{1}(0)=s_{2}(0)=1 / 2, k_{1}(0)=0.3, k_{2}(0)=k_{1}(0) / 4$.

Indeed, from the proof of Lemma 4.1, we know that the coincidence point $R^{*}$ must lie above the $\frac{a_{1}}{a_{2}}$ threshold. This means that the equlibirum quotient exceeds the quotient of coefficients

$$
\frac{k_{1}^{*}}{k_{2}^{*}}=R^{*}>\frac{a_{1}}{a_{2}} .
$$

Again, the eigenvalues of the Jacobian at the equilibrium point corresponding to $R^{*}>\frac{a_{1}}{a_{2}}$ have negative real part and hence the equilibrium point is asymptotically stable. We omit the details of this technical computation, but in order to strike a balance between the abstract and the concrete level, we mention that given the realistic values

$$
a_{1}=0.18, a_{2}=0.15, \alpha=1 / 3, b=0.06, c=0.1
$$

for the parameters, one obtains

$$
R^{*}=2.208, \quad k_{1}^{*}=2.773 \quad \text { and } \quad k_{2}^{*}=1.256
$$

such that the eigenvalues of the Jacobi matrix are all negative

$$
\lambda_{1,2,3}=-4.081,-0.076 \text { and }-0.019 .
$$

\section{Conclusions}

In order to describe the economic implications of a prolonged military rivalry, we have constructed a nonlinear dynamical model that merges the classical Richardson arms race evolution with economic growth in the sense of Solow's pioneering work.

The ensuing nonstandard model turns out to be well-posed and in accordance with both political and economic intuitions. More precisely, when considering different levels of technology for the two competing powers, the model predicts that in the long 
run, due to nonlinear effects, the sizes of the two economies will be separated by a gap that exceeds the technology gap.

The reality of Cold War dynamics has been far more complex than the relatively simple model that we propose can describe. Many extensions are possible and, actually desirable. The most natural extension would be to consider the augmented human capital version of the Solow-Swan model due to Mankiw, Romer and Weil [11] not the Solow-Swan economic growth model itself.

Furthermore, the Cold War arms race is just a prototype for more general economic rivalry phenomena. Trade or economic wars provide very interesting challenges form a modeling perspective.

\section{References}

[1] Acemoglu, D., Introduction to Modern Economic Growth, Princeton University Press, 2011.

[2] Barbu, V., Differential Equations, Springer Undergraduate Mathematics Series, Springer, Cham, 2016. Translated from the 1985 Romanian original by Liviu Nicolaescu.

[3] Barro, R.J., Sala-i Martin, X., Economic Growth, MIT Press, 2004.

[4] Bueno de Mesquita, B., Lalman, D., Arms races and the opportunity for peace, Formal Analysis in International Relations, 76(1988), 263-283.

[5] Dawkins, R., Krebs, J.R., Arms races between and within species, Proceedings of the Royal Society of London, Series B, Biological Sciences, 205(1161)(1979), 489-511.

[6] Hill, W.W., Several sequential augmentations of Richardson's arms race model, Math. Comput. Modelling, 6(8-9)(1992).

[7] Intriljgator, M.D., Brito, D.L., Arms races, Defence and Peace Economics, 11(2000), no. $1,45-54$.

[8] Krabs, W., Dynamische Systeme: Steuerbarkeit und Chaotisches Verhalten, B. G. Teubner, Stuttgart, 1998.

[9] Larrosa, J.M.C., Arms build-up and arms race in optimal economic growth, Int. J. Econ. Theory, 12(2016), no. 2, 167-182.

[10] Majeski, S.J., Arms races as iterated prisoner's dilemma games, Math. Social Sci., 7(3)(1984), 253-266.

[11] Mankiw, N.G., Romer, D., Weil, D.N., A contribution to the empirics of economic growth, The Quarterly Journal of Economics, 107(1992), no. 2, 407-437.

[12] Richardson, L.F., Arms and Insecurity: A Mathematical Study of the Causes and Origins of War, Edited by Nicolas Rashevsky and Ernesto Trucco, The Boxwood Press, Pittsburgh, Pa.; Quadrangle Books, Chicago, Ill., 1960.

[13] Richardson, L.F., Statistics of Deadly Quarrels, Boxwood Press, 1960.

[14] Solow, R.M., A contribution to the theory of economic growth, The Quarterly Journal of Economics, 70(1956), no. 1, 65-94.

[15] Swan, T.W., Economic growth and capital accumulation, Economic Record, 32(1956), no. $2,334-361$. 
Daniel Metz

NTT DATA Romania

19-21, Constanţa Street, 400158 Cluj-Napoca, Romania

e-mail: Daniel.Metz@nttdata.ro

Adrian Viorel

"Babeş-Bolyai" University

Faculty of Mathematics and Computer Sciences

1, Kogălniceanu Street, 400084 Cluj-Napoca, Romania

e-mail: adrian.viorel@math.ubbcluj.ro 